\title{
A new formulation of internal forces for non-linear hypoelastic constitutive models verifying conservation laws
}

\author{
Ludovic NOELS ${ }^{1}$, Laurent STAINIER ${ }^{1}$, Jean-Philippe PONTHOT ${ }^{1}$ and Jérôme BONINI ${ }^{2}$. \\ ${ }^{1}$ LTAS-Milieux Continus et Thermomécanique, University of Liège, Chemin des Chevreuils 1, 4000 Liège, Belgium \\ ${ }^{2}$ SNECMA Moteurs - Engineering Division, centre de Villaroche, 77550 Moissy-Cramayel, France.
}

Second MIT Conference on Fluid and Solid Mechanics K.J. Bathe (ed)

17-20 June 2003 Boston, USA, Vol. 1 pages 527-531. 


\title{
A new formulation of internal forces for non-linear hypoelastic constitutive models verifying conservation laws
}

\author{
L. Noels ${ }^{\mathrm{a}, *}$, L. Stainier ${ }^{\mathrm{a}}$, J.-P. Ponthot ${ }^{\mathrm{a}}$, J. Bonini ${ }^{\mathrm{b}}$ \\ ${ }^{a}$ University of Liège, LTAS-Thermomechanics, Chemin des Chevreuils 1, Bât. B52/3, 4000 Liège 1, Belgium \\ ${ }^{b}$ SNECMA-Moteurs, Engineering Division, centre de Villaroche, 77550 Moissy-Cramayel, France
}

\begin{abstract}
This paper proposes a new formulation of the internal forces for hypoelastic constitutive models ensuring that the elastic work of deformation can be restored by the scheme. Moreover, we demonstrate that the work of the plastic deformation is positive and consistent with the material model.
\end{abstract}

Keywords: Energy-momentum; Conserving algorithm; Dynamics; Hypoelastic material; Large strain plasticity; Constitutive models

\section{Introduction}

A new kind of integration algorithms for dynamics has recently appeared, which verifies the mechanical laws of conservation (i.e. conservation of the linear and angular momentum and of the total energy) and which remains stable in the non-linear range. The first algorithm verifying these properties was described by Simo and Tarnow [1]. This algorithm was called Energy Momentum Conserving Algorithm or EMCA. It consists in a mid-point scheme with an adequate evaluation of the internal forces, and was derived for a Saint Venant-Kirchhoff hyperelastic material. Laursen [2] generalized this approach to other hyperelastic models. His method involves resolving iteratively a new equation for each material point in order to compute the adequate Piola-Kirchhoff stress tensor. Another solution, that avoids this iterative procedure and leads to a general formulation of the Piola-Kirchhoff stress tensor, was proposed by Gonzalez [3]. The EMCA was recently extended to dynamic finite deformation plasticity by Meng and Laursen [4].

To our knowledge, the EMCA conserving algorithm was never extended to hypoelastic materials. This paper proposes such an extension, through a new expression of the internal forces, which verifies the conservation laws. Moreover, we prove that this expression remains consistent when plastic deformations occurs. A numerical example illustrates the good accuracy of the proposed formulation.

* Corresponding author. Tel.: +32436691 85; Fax: +324366

91 41; E-mail: L.Noels@ulg.ac.be

\section{The hypoelastic model}

Let the configuration $n$ be the configuration computed after $n$ time steps (i.e. at time $t_{n}$ ). Let $x^{n}$ be the deformation mapping (coordinates) in the configuration $n$, and let $x^{n^{\xi}}$ be the nodal coordinates for the node $\xi$. With $\varphi^{\xi}$ the shape function linked to node $\xi$, it comes (Einstein's notations are used)

$x=\varphi^{\xi} x^{\xi}$

The gradient of deformation (two point tensor) $\boldsymbol{F}$ between configurations $m$ and $n$ is indicated by $\boldsymbol{F}_{m}^{n}$. The tensor $\boldsymbol{f}$ represents $\boldsymbol{F}^{-1}$. This gradient tensor can be decomposed into a rotation tensor $\boldsymbol{R}$ and a symmetric deformation tensor $\boldsymbol{U}$. The determinant of $\boldsymbol{F}_{m}^{n}$ is denoted by the scalar $J_{m}^{n}$. The natural strain tensor $\boldsymbol{E}_{m}^{n}$, the Green-Lagrange strain tensor $\boldsymbol{G L}_{m}^{n}$, and the Almansi strain tensor $\boldsymbol{A}_{m}^{n}$ are respectively defined as

$\boldsymbol{E}_{m}^{n}=\frac{1}{2} \ln \left[\boldsymbol{F}_{m}^{n T} \boldsymbol{F}_{m}^{n}\right]$

$\boldsymbol{G} \boldsymbol{L}_{m}^{n}=\frac{1}{2}\left[\boldsymbol{F}_{m}^{n T} \boldsymbol{F}_{m}^{n}-\boldsymbol{I}\right]$

$\boldsymbol{A}_{m}^{n}=\frac{1}{2}\left[\boldsymbol{I}-\boldsymbol{f}_{m}^{n T} \boldsymbol{f}_{m}^{n}\right]$

The Cauchy stress tensor is evaluated in the configuration $n$ and is referred to as $\boldsymbol{\Sigma}^{n}$. For the hypoelastic constitutive laws, the Cauchy stress tensor is computed from a stress increment deduced from the incremental natural strain tensor. With $\mathcal{H}$ the Hooke fourth order tensor and with the operation $\mathcal{H}: \boldsymbol{E}$ defined by $\mathcal{H}_{i j k l} \boldsymbol{E}_{k l}$, the final rotation scheme 
$[5,6]$ is defined by the following relation

$\boldsymbol{\Sigma}^{n+1}=\boldsymbol{R}_{n}^{n+1}\left[\boldsymbol{\Sigma}^{n}+\mathcal{H}: \boldsymbol{E}_{n}^{n+1}-\boldsymbol{s}^{c}\left(\varepsilon^{p}\right)\right] \boldsymbol{R}_{n}^{n+1^{T}}$

In this relation $\boldsymbol{s}^{c}$, resulting from the plastic deformation, has a trace equal to zero and is given by the radial return mapping (for isotropic materials with $J 2$-plasticity) [6]. The scalar $\varepsilon^{p}$ is the equivalent plastic strain.

\section{The energy-momentum conserving scheme}

\subsection{The mid-point scheme [1]}

For an integration from time $t_{n}$ to time $t_{n}+\Delta t=t_{n+1}$, if the variable $z$ represents the position or the velocity, it leads

$$
\begin{aligned}
\dot{z}^{n+1 / 2} & =\frac{z^{n+1}-z^{n}}{\Delta t} \\
& =\frac{\dot{z}^{n+1}+\dot{z}^{n}}{2}
\end{aligned}
$$

The balance law for node $\xi$ is

$M^{\xi \mu}\left[\ddot{x}^{n+1 / 2}\right]^{\mu}=\left[F_{\mathrm{ext}}^{n+1 / 2}-F_{\mathrm{int}}^{n+1 / 2}\right]^{\xi}$

where $M$ is the mass matrix, $F_{\text {ext }}^{n+1 / 2}$ and $F_{\text {int }}^{n+1 / 2}$ are respectively the external and internal forces evaluated in configuration $n+\frac{1}{2}$. This expression indirectly depends on the positions in configuration $n+1$ (i.e. $x^{n+1}$ ). The goal of this work is to evaluate the expression of the internal forces in configuration $n+\frac{1}{2}$ for hypoelastic models. The system of Eqs. (4) and (5) is resolved iteratively.

\subsection{The conservation conditions}

Eq. (5) has to verify the conservation of the linear momentum:

$\sum_{\xi}\left[F_{\text {int }}^{n+1 / 2}\right]^{\xi}=0$,

the conservation of the angular momentum:

$\left[x^{n+1 / 2 \xi} \wedge F_{\text {int }}^{n+1 / 2 \xi}\right]=0$,

and the conservation of the energy [1]:

$\left[F_{\mathrm{int}}^{n+1 / 2}\right]^{\xi} *\left[x^{n+1}-x^{n}\right]^{\xi}=W_{\mathrm{int}}^{n+1}-W_{\mathrm{int}}^{n}+\Delta_{\mathrm{int}}$

where $W_{\text {int }}$ and $\Delta_{\text {int }}$ are respectively the internal energy and the plastic dissipation. The operator $\wedge$ denotes the vector product and the operator $*$ denotes the scalar product.

\section{Internal forces expression for the hypoelastic model}

Let $D$ be the derivative of the shape function in the reference configuration $D^{\xi}=\partial \varphi^{\xi} / \partial x^{0}$. Then, the following expression is proposed

$$
\begin{aligned}
& {\left[F_{\text {int }}^{n+1 / 2}\right]^{\xi}=\frac{1}{2}\left[F_{\text {int }}^{*}+F_{\text {int }}^{* *}\right]^{\xi}} \\
& {\left[F_{\text {int }}^{*}\right]^{\xi}=\frac{1}{2} \int_{V_{0}}\left\{\left[\boldsymbol{I}+\boldsymbol{F}_{n}^{n+1}\right] \boldsymbol{\Sigma}^{n} \boldsymbol{f}_{0}^{n T} D^{\xi} J_{0}^{n}\right\} \mathrm{d} V_{0}} \\
& +\frac{1}{2} \int_{V_{0}}\left\{\left[\boldsymbol{I}+\boldsymbol{F}_{n}^{n+1}\right] \frac{\boldsymbol{c}^{*}: \boldsymbol{\Sigma}^{n}}{\boldsymbol{G} \boldsymbol{L}_{n}^{n+1}: \boldsymbol{G L}_{n}^{n+1}} \boldsymbol{G} \boldsymbol{L}_{n}^{n+1} \boldsymbol{f}_{0}^{n T} D^{\xi} J_{0}^{n}\right\} \mathrm{d} V_{0} \\
& {\left[F_{\text {int }}^{* *}\right]^{\xi}=\frac{1}{2} \int_{V_{0}}\left\{\left[\boldsymbol{I}+\boldsymbol{f}_{n}^{n+1}\right] \boldsymbol{\Sigma}^{n+1} \boldsymbol{f}_{0}^{n+1^{T}} D^{\xi} J_{0}^{n+1}\right\} d V_{0}} \\
& +\frac{1}{2} \int_{V_{0}}\left\{\left[\boldsymbol{I}+\boldsymbol{f}_{n}^{n+1}\right] \frac{\boldsymbol{c}^{* *}: \boldsymbol{\Sigma}^{n+1}}{\boldsymbol{A}_{n}^{n+1}: \boldsymbol{A}_{n}^{n+1}} \boldsymbol{A}_{n}^{n+1} \boldsymbol{f}_{0}^{n+1} D^{\xi} J_{0}^{n+1}\right\} \mathrm{d} V_{0}
\end{aligned}
$$

where $c^{*}$ and $c^{* *}$ are tensors yet to be determined. This expression can be shown to conserve the properties of the final rotation scheme and of the radial return mapping scheme [7].

\subsection{Verification of conservation laws}

The conservation of the linear momentum (6) is directly reached using the properties of the shape functions $\left(\sum_{\xi} D_{K}^{\xi}=0\right)$. The conservation of the angular momentum (7) is a consequence of the symmetry of the Cauchy stress tensor [7].

Since no potential can be defined for a hypoelastic material model we define a loading-unloading cycle, from configuration 1 to 3 , where the initial Cauchy stress tensor corresponds to the final Cauchy stress tensor for any rotation $Q$

$\Sigma^{3}=Q \Sigma^{1} Q^{T}$

During the loading phase from configuration 1 to 2 , plastic deformations occur. During the unloading phase from configuration 2 to 3 , there is no subsequent plastic deformation. Then Eq. (9) is consistent with Drucker's Postulate if the reversible work of the first step is recovered during the second step. Therefore, the energy balance between configurations 1 and 3 can be expressed as

$\left[F_{\mathrm{int}}^{3 / 2}\right]^{\xi} *\left[x^{2}-x^{1}\right]^{\xi}+\left[F_{\mathrm{int}}^{5 / 2}\right]^{\xi} *\left[x^{3}-x^{2}\right]^{\xi}=\Delta_{\mathrm{int}}$

Let $\boldsymbol{E}^{\mathrm{el}^{2}}$ be the elastic natural strain tensor defined such that

$\mathcal{H}: \boldsymbol{E}_{1}^{\mathrm{el}^{2}} \equiv \mathcal{H}: \boldsymbol{E}_{1}^{2}-\boldsymbol{s}_{1}^{c 2}$

Then we define $\boldsymbol{U}_{1}^{\mathrm{el}^{2}}$ a symmetric tensor such that

$\boldsymbol{E}_{1}^{\mathrm{el}^{2}} \equiv \frac{1}{2} \ln \left[\boldsymbol{U}_{1}^{\mathrm{el}^{2} \boldsymbol{U}^{\mathrm{el}}{ }^{2}}\right]$

The elastic and plastic Green-Lagrange strain tensor $\left(\boldsymbol{G} \boldsymbol{L}_{1}^{\mathrm{e}^{2}}\right.$ and $\boldsymbol{G} \boldsymbol{L}^{\mathrm{pl}}{ }_{1}^{2}$ ), and the elastic and plastic Almansi strain tensor 
$\left(\boldsymbol{A}_{1}^{\mathrm{el}^{2}}\right.$ and $\left.\boldsymbol{A}_{1}^{\mathrm{pl}^{2}}\right)$ are defined from $\boldsymbol{U}_{1}^{\mathrm{el}^{2}}$ as

$\boldsymbol{G}^{\mathrm{el}^{2}}{ }_{1}^{2} \equiv \frac{1}{2}\left[\boldsymbol{U}_{1}^{\mathrm{el}^{2}} \boldsymbol{U}_{1}^{\mathrm{el}^{2}}-\boldsymbol{I}\right]$

$\boldsymbol{G L}_{1}^{\mathrm{pl}^{2}} \equiv \boldsymbol{G} \boldsymbol{L}_{1}^{2}-\boldsymbol{G L}_{1}^{\mathrm{el}^{2}}$

$\boldsymbol{A}_{1}^{\mathrm{el}^{2}} \equiv \frac{1}{2} \boldsymbol{R}_{1}^{2}\left[\boldsymbol{I}-\boldsymbol{U}_{1}^{\mathrm{el}^{2-1}} \boldsymbol{U}_{1}^{\mathrm{el}^{2-1}}\right] \boldsymbol{R}_{1}^{2^{T}}$

$\boldsymbol{A}_{1}^{\mathrm{pl}^{2}} \equiv \boldsymbol{A}_{1}^{2}-\boldsymbol{A}_{1}^{\mathrm{el}^{2}}$

Therefore, since the tensors $\boldsymbol{c}^{*}$ and $\boldsymbol{c}^{* *}$ can be taken equal to zero when no plastic deformation occurs [7], we can rewrite Eq. (11) as [7]:

$$
\begin{aligned}
\Delta_{\text {int }}=\frac{1}{2} \int_{V_{0}}\{ & {\left[\boldsymbol{G} \boldsymbol{L}_{1}^{\mathrm{pl}^{2}}+\boldsymbol{c}^{*}\right]: \boldsymbol{\Sigma}^{1} J^{0,1} } \\
+ & {\left.\left[\boldsymbol{A}_{1}^{\mathrm{pl}^{2}}+\boldsymbol{c}^{* *}\right]: \boldsymbol{\Sigma}^{2} J^{0,2}\right\} \mathrm{d} V_{0} }
\end{aligned}
$$

If $D_{\text {int }}$ is the analytical expression of the volumic dissipation during transformation from configuration 1 to 3 , the following expression of the tensors

$\boldsymbol{c}^{*}=\frac{D_{\mathrm{int}}}{\boldsymbol{\Sigma}^{1} J_{0}^{1}: \boldsymbol{\Sigma}^{2} J_{0}^{2}} \boldsymbol{\Sigma}^{2} J_{0}^{2}-\boldsymbol{G} \boldsymbol{L}_{1}^{2}+\boldsymbol{G} \boldsymbol{L}_{1}^{\mathrm{el}}$

$\boldsymbol{c}^{* *}=\frac{D_{\mathrm{int}}}{\Sigma^{1} J_{0}^{1}: \Sigma^{2} J_{0}^{2}} \boldsymbol{\Sigma}^{1} J_{0}^{1}-\boldsymbol{A}_{1}^{2}+\boldsymbol{A}_{1}^{\mathrm{el}^{2}}$

leads to

$\Delta_{\text {int }}=\int_{V_{0}}\left\{D_{\text {int }}\right\} \mathrm{d} V_{0}$

which is consistent with the energy conservation laws [7]. Moreover, the magnitude of the tensors $c^{*}$ and $c^{* *}$ is of second order in the increment of the plastic deformation [7] and thus can be omitted if the time step size is small enough.

\section{Numerical example}

Results obtained with the conservative algorithm (EMCA) are compared with the results obtained with the Newmark algorithm (NMK) (first Newmark parameter $\beta$ equal to 0.25 and second Newmark parameter $\gamma$ equal to $0.5)$.

\subsection{Taylor's bar problem}

This example was first simulated with a conservative algorithm for a hyperelastic Saint Venant-Kirchhoff material by Meng and Laursen [4]. It consists in a cylindrical bar (Table 1), discretized by 576 elements. It has an initial velocity $\dot{x}_{0}$. The time step size is equal to $0.1 \mu \mathrm{s}$. Fig. 1 represents the evolution of the total energy (since no potential exists the total energy is the work of the internal and of the inertial forces, internal dissipation included). Fig. 2 represents the evolution of the internal dissipation. The final plastic strains

Table 1

Properties of the Taylor bar problem

\begin{tabular}{ll}
\hline Property & Value \\
\hline External diameter & $d_{e}=6.4 \mathrm{~mm}$ \\
Length & $l=32.4 \mathrm{~mm}$ \\
Density & $\rho=8930 \mathrm{~kg} / \mathrm{m}^{3}$ \\
Young modulus & $E=117 \times 10^{9} \mathrm{~N} / \mathrm{m}^{2}$ \\
Poisson ratio & $v=0.35$ \\
Yield stress & $\sigma_{0}=400 \mathrm{~N} / \mathrm{mm}^{2}$ \\
Hardening parameter & $h=100 \mathrm{~N} / \mathrm{mm}^{2}$ \\
Initial velocity & $\dot{x}_{0}=227 \mathrm{~m} / \mathrm{s}$ \\
\hline
\end{tabular}

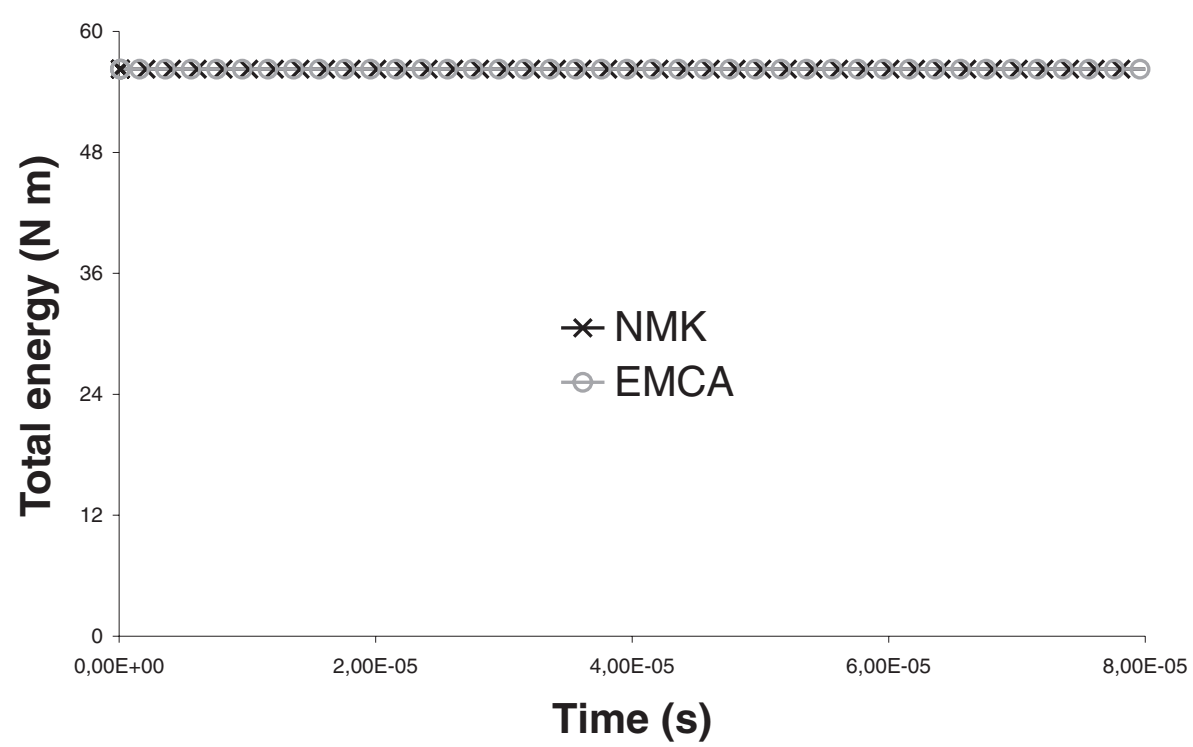

Fig. 1. Evolution of the total energy for the Taylor bar problem. 


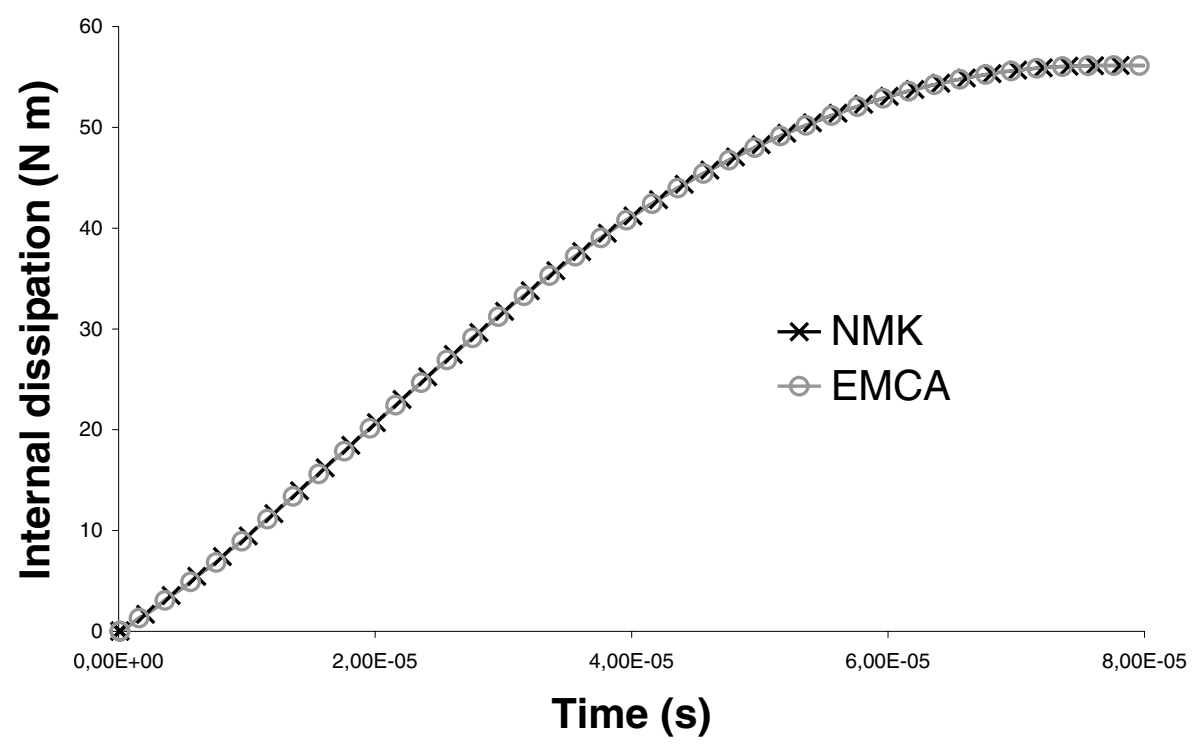

Fig. 2. Evolution of the total internal dissipation for the Taylor bar problem.

\section{NMK}

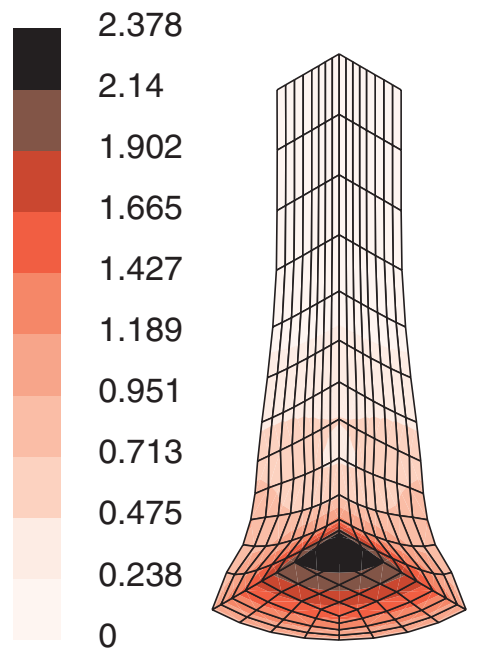

\section{EMCA}

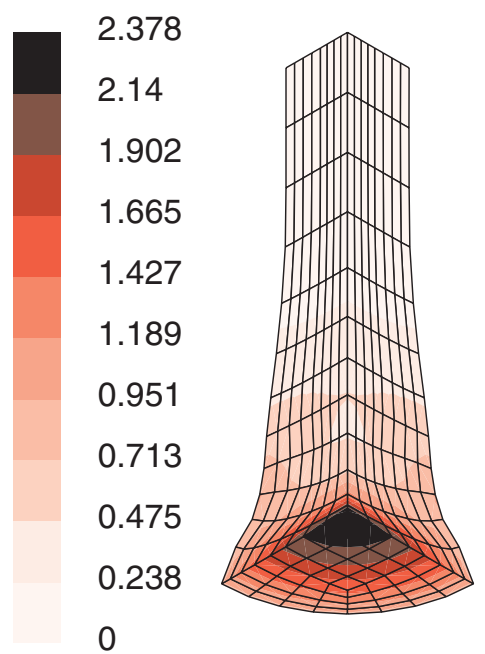

Fig. 3. Equivalent plastic strain for the Taylor bar problem after $80 \mu \mathrm{s}$.

are illustrated in Fig. 3. Results obtained with both schemes are similar. The Newmark scheme requires 1951 iterations and is $2.5 \%$ more expensive than the conservative scheme (1904 iterations).

\section{Conclusions}

A new expression of the internal forces for a hypoelastic material was presented. When used with the mid-point scheme, this expression leads to a energy-momentum conservative scheme. Moreover, the internal dissipation, resulting from the plastic deformation is consistent with the laws of thermodynamic. The solution obtained with this conservative scheme was compared with the results obtained with the Newmark scheme for the Taylor bar impact, leading to similar results. Note that the EMCA is expected to show an improved performance for larger time steps, when the second order terms gain importance, but this is part of a future work. 


\section{Acknowledgements}

L. Noels and L. Stainier wish to acknowledge support from the Belgian National Fund for Scientific Research (F.N.R.S.).

\section{References}

[1] Simo JC, Tarnow N. The discrete energy-momentum method. Conserving algorithms for nonlinear elastodynamics. ZAMP 1992;43:757-792.

[2] Laursen T, Meng X. A new solution procedure for application of energy-conserving algorithms to general constitutive models in nonlinear elastodynamics. Comput Methods Appl Mechanics Engrg 2001;190:6309-6322.
[3] Gonzalez O. Exact energy and momentum conserving algorithms for general models in nonlinear elasticity. Comput Methods Appl Mechanics Engrg 2000;190:1763-1783.

[4] Meng X, Laursen T. Energy consistent algorithms for dynamic finite deformation plasticity. Comput Methods Appl Mechanics Engrg 2001;191:1639-1675.

[5] Nagtegaal J, Veldpaus F. On the implementation of finite strain plasticity equations in a numerical model. In: Pittman et al. (Eds), Numerical Analysis of Forming Processes, John Wiley, 1984.

[6] Ponthot JP. Unified stress update algorithms for the numerical simulation of large deformation elasto-plastic and elastoviscoplastic processes. Int J Plasticity 2002;18:91-126.

[7] Noels L, Stainier L, Ponthot JP. Energy-momentum conserving algorithm for non-linear hypoelastic constitutive models. Int J Numer Methods Engrg 2003; submitted. 